\title{
NEURODESARROLLO INFANTIL: CARACTERÍSTICAS NORMALES Y SIGNOS DE ALARMA EN EL NIÑO MENOR DE CINCO AÑOS
}

\author{
María del Pilar Medina Alva1,2,a, Inés Caro Kahnn,1,a, Pamela Muñoz Huerta ${ }^{1,3, a}$, Janette Leyva Sánchez ${ }^{1,4, a}$, \\ José Moreno Calixto ${ }^{1,5, a}$, Sarah María Vega Sánchez $z^{1, b}$
}

\begin{abstract}
RESUMEN
El desarrollo del sistema nervioso es un proceso complejo que tiene como resultado la maduración de las estructuras, la adquisición de habilidades y, finalmente, la formación del individuo como persona única. La presente revisión recoge información acerca de las principales características de los procesos de desarrollo cerebral, las características del desarrollo neurológico normal en las diferentes áreas: motora gruesa y fina, lenguaje, sensorial y socialización; se acompaña también de una descripción de las principales alteraciones en el desarrollo, identificables en la consulta diaria del pediatra. Nuestro objetivo es reforzar el conocimiento en esta área clave de la evaluación del niño menor de cinco años para detectar problemas con la debida antelación para su intervención oportuna.
\end{abstract}

Palabras clave: Desarrollo infantil; Neurología; Pediatría (fuente: DeCS BIREME).

\section{CHILD NEURODEVELOPMENT: NORMAL CHARACTERISTICS AND WARNING SIGNS IN CHILDREN UNDER FIVE YEARS}

\begin{abstract}
The development of the nervous system is a complex process that results in the maturation of structures, the acquisition of skills and finally the formation of the individual as a unique person. This review contains information about the main characteristics of the processes of brain development, the characteristics of normal neurological development in different areas: gross and fine motor, language, sensory and socialization; and it is also accompanied by a description of the major changes in the development, identifiable in the daily clinical work of pediatricians. Our goal is to enhance knowledge in this key area of assessment of early childhood to detect problems sufficiently in advance for their timely intervention.
\end{abstract}

Key words: Child development; Neurology; Pediatrics (source: MeSH NLM).

\section{INTRODUCCIÓN}

El desarrollo infantil, su seguimiento de manera regular y periódica y la detección precoz de signos de alarma que señalen alteraciones en detrimento de su evolución normal, tienen repercusión crucial para lograr el máximo potencial de las capacidades y habilidades de cada ser humano y de la sociedad en su conjunto.

Resulta, entonces, fundamental que el pediatra y todo médico o profesional de la salud que atiende niños, conozca a profundidad las características propias del neurodesarrollo en las diferentes etapas de la vida del ser humano y en sus diferentes manifestaciones; no solo motoras gruesas, que son las que a menudo suelen priorizarse en los controles de crecimiento y desarrollo, sino en otras áreas como la motora fina, sensorial, lenguaje y socioemocional.

En la actualidad, un aspecto que ha cobrado mucha importancia por su sólida base científica es el hecho que el neurodesarrollo exitoso tiene estrecha relación no solo con la genética, sino también con el ambiente de estimulación y afectividad que rodea al niño, los cuales influyen decisivamente en la mayor producción de sinapsis neuronales, lo cual implica, a su vez, en la mayor integración de las funciones cerebrales.

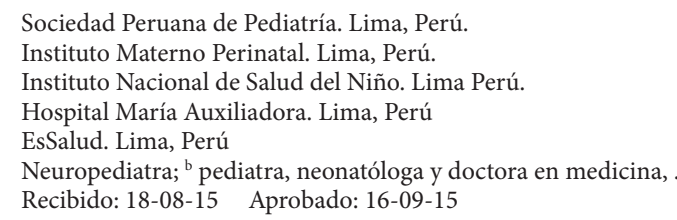

Citar como: Medina Alva MDP, Caro-Kahn I, Muñoz Huerta P, Leyva Sánchez J, Moreno Calixto J, Vega Sánchez SM. Neurodesarrollo infantil: características normales y signos de alarma en el niño menor de cinco años. Rev Peru Med Exp Salud Publica. 2015;32(3):565-73. 
También la nutrición de calidad y la lactancia materna muestran influencia clave para el desarrollo y resultados futuros de mejor productividad y calidad de vida; estudios recientes como, por ejemplo, el de Victora et al. (1), muestran una asociación positiva de dosis respuesta (duración) entre la lactancia materna y el cociente intelectual, los logros educacionales e ingresos a los 30 años de edad.

Por otro lado, estudios de revisión de la microbioma intestinal (2), muestran que los microbios que habitan el tracto gastrointestinal forman un ecosistema específico, influido en cada ser humano por diversos factores, como el tipo de parto, la edad gestacional, la nutrición y el uso temprano de antibióticos. Este ecosistema también tiene una ligazón importante con el cerebro en desarrollo, por lo cual, mediante estudios futuros, se espera entender esta relación que podría ser de ayuda para tratar varias enfermedades como el autismo, la esquizofrenia y la ansiedad. Motivo por el cual, un equipo de neuropediatras integrantes del Capítulo de Neurología Pediátrica de la Sociedad Peruana de Pediatría presentamos una visión global de las características de la evolución del neurodesarrollo infantil normal, sus alteraciones más frecuentes y los principales signos de alarma.

\section{GENERALIDADES SOBRE EL DESARROLLO NORMAL DEL CEREBRO}

El neurodesarrollo se da a través de un proceso dinámico de interacción entre el niño y el medio que lo rodea; como resultado, se obtiene la maduración del sistema nervioso con el consiguiente desarrollo de las funciones cerebrales y, a la vez, la formación de la personalidad. El desarrollo del cerebro es un proceso muy complejo y preciso que inicia muy temprano en la vida y continúa varios años después del nacimiento. Existen periodos críticos para el desarrollo cerebral normal, siendo los principales la vida intrauterina y el primer año de vida. Podemos resumir las etapas del desarrollo del cerebro en estas cuatro: proliferación neuronal, migración, organización y laminación del cerebro, y mielinización (3). No son etapas consecutivas, se van superponiendo y pueden ser afectadas simultáneamente si existe algún agente externo o interno presente en el medio. Un ejemplo es el consumo de alcohol en la madre gestante, o la desnutrición del niño menor de dos años. Ambos procesos pueden afectar más de una fase en forma permanente.

La proliferación de las neuronas es un proceso que ocurre en la primera mitad de la gestación. A través de este proceso se da origen a los cien mil millones de neuronas que el cerebro posee. Todas las neuronas deben desplazarse a su lugar final en la corteza durante el proceso llamado migración, este segundo proceso se da de adentro hacia afuera, es decir, desde la parte más profunda del cerebro, donde nacen las neuronas, hasta la corteza o borde externo. Se trata de un proceso muy preciso, y el momento más importante ocurre en el segundo trimestre del embarazo. Puede ser afectado por la exposición fetal a medicamentos, infecciones, tóxicos, desnutrición y estrés materno, entre otros, y producirse malformaciones cerebrales importantes como consecuencia de estos eventos, conocidas como trastornos de migración neuronal.

Después de las 25 semanas posconcepcionales, la reproducción de nuevas neuronas es excepcional. Sin embargo, el peso del cerebro se triplica después que la fase de proliferación ha terminado. Este sorprendente incremento en peso y volumen obedece a la aparición de millones de conexiones sinápticas entre las neuronas y a la arborización, resultado de la aparición de dendritas. Se estima que cada neurona puede llegar a tener entre 7000 y 10000 sinapsis, las cuales, posteriormente, podrán ser modeladas según la exposición a factores externos e internos y experiencias que modifican su conformación en forma permanente ${ }^{(4)}$. El último proceso en iniciarse es la mielinización, en el que los axones de las neuronas se recubren de mielina para mejorar la velocidad de transmisión de los impulsos nerviosos. Este es un proceso crítico que inicia cerca del nacimiento. Sin embargo, puede verse severamente alterado en los primeros meses de vida como consecuencia de la falta de nutrientes, el hipotiroidismo, la anemia y la falta de una adecuada estimulación en el niño pequeño.

\section{EVALUACIÓN DEL NEURODESARROLLO}

La evaluación de los hitos del desarrollo en el niño permite estimar que el desarrollo cerebral está ocurriendo dentro de un marco apropiado, por tanto, es muy importante conocer los parámetros mínimos de evaluación del desarrollo para cada edad. Para ello, nos podemos ayudar del carné de atención del niño o de algunas escalas previamente validadas. Recordemos que los hitos del desarrollo tienen un amplio margen de variabilidad normal, y es más importante establecer que se está logrando una secuencia adecuada de eventos en el tiempo, que fijarse en un determinado logro puntual. Como ejemplo, podemos poner el hito del caminar, que en promedio es logrado por el niño alrededor del año de vida. Sin embargo, según la OMS, un niño puede empezar a caminar entre los 9 y 17 meses, siendo esta variabilidad normal. En el niño que demora en caminar debemos determinar si los pasos previos (sedestación, gateo, marcha con apoyo) se han logrado a tiempo, o si todo el proceso motor grueso está demorado también. En este último caso, el diagnóstico del niño 
es de retraso en el desarrollo y como tal deberá ser evaluado. Debemos recordar, además, que la pérdida de habilidades previamente adquiridas, o regresión del desarrollo, siempre implica una alerta para que el niño sea derivado inmediatamente para su evaluación complementaria ${ }^{(5)}$.

Otro punto muy importante en la evaluación del neurodesarrollo es determinar si el tamaño de la cabeza corresponde al apropiado para la edad del paciente. El perímetro cefálico es una medida de referencia muy sencilla para realizarse en cualquier consultorio o ambiente que nos permite estimar de forma rápida si los procesos de desarrollo del cerebro se han dado en forma adecuada. El hallazgo de microcefalia o macrocefalia también es motivo de atención.

\section{SIGNOS TEMPRANOS DE ALARMA}

Existen algunos parámetros que nos permiten identificar alteraciones relevantes en el neurodesarrollo, como son la falla en el progreso del desarrollo a una edad determinada, el desarrollo asimétrico del movimiento, tono o reflejos, la pérdida de habilidades previamente adquiridas, y la pobreza de interacción social y psicoafectividad. Algunas alteraciones específicas que pueden hallarse desde edades muy tempranas son de tipo motor: pulgar cautivo, dominancia establecida antes del primer año, persistencia de reflejos primitivos, anormalidades persistentes del tono muscular y demora en la aparición de reflejos. Otro aspecto de interés mayor en los últimos años es el desarrollo social, con el objetivo de la identificación temprana de trastornos del espectro autista. El desarrollo sensorial debe ser evaluado en el niño muy pequeño, quien debe ser capaz de responder a estímulos visuales y auditivos en forma adecuada en el primer trimestre de vida. Finalmente, una curva anormal de crecimiento craneal es otro signo de alarma relevante.

\section{DESARROLLO MOTOR}

El desarrollo motor involucra la adquisición progresiva de habilidades motoras que permiten mantener un adecuado control postural, desplazamiento y destreza manual. Para ello, se requiere la aparición y desaparición de los reflejos controlados por los niveles inferiores del sistema nervioso central (SNC) que permiten respuestas posturales y motoras funcionales y voluntarias. Asimismo, el control postural surge de un compleja interacción entre el sistema musculoesquelético y nervioso, denominados en conjunto sistema de control postural. El entorno o medioambiente cumple una función fundamental. Existen factores reguladores del desarrollo motor como los de tipo endógeno o no modificables que son los genéticos y neurohormonales, y los de tipo exógeno o modificables donde se encuentran la nutrición, el estado de salud, los factores psicológicos y los factores socioeconómicos.

El desarrollo motor grueso se produce en sentido cefalocaudal, y se refiere a los cambios de posición del cuerpo y la capacidad de control que se tiene sobre este para mantener el equilibrio, la postura y el movimiento, con lo cual se logra controlar la cabeza, sentarse sin apoyo, gatear, caminar, saltar, correr, subir escaleras, etc. ${ }^{(6)}$. El desarrollo motor fino se produce en sentido próximo distal, y está relacionado con el uso de las partes individuales del cuerpo, como las manos; lo cual requiere de la coordinación óculomanual para poder realizar actividades como coger juguetes, manipularlos, agitar objetos, dar palmadas, tapar o destapar objetos, agarrar cosas muy pequeñas, enroscar, hasta llegar a niveles de mayor complejidad como escribir ${ }^{(6)}$.

Si bien es cierto, existen una serie de escalas para valorar el desarrollo psicomotor, se ha intentado sistematizar dicha evaluación en periodos trimestrales y semestrales (Tabla 1).

Tabla 1. Hitos del desarrollo motor del niño

\begin{tabular}{|c|c|c|}
\hline Edad & Motor grueso & Motor fino \\
\hline 3 meses & Control cefálico & $\begin{array}{l}\text { Coge objetos en línea } \\
\text { media }\end{array}$ \\
\hline 6 meses & $\begin{array}{l}\text { Se mantiene } \\
\text { sentado }\end{array}$ & $\begin{array}{l}\text { Transfiere de una mano } \\
\text { a otra }\end{array}$ \\
\hline 9 meses & $\begin{array}{l}\text { Se sienta por sí } \\
\text { solo y gatea }\end{array}$ & Pinza gruesa o inmadura \\
\hline 12 meses & Camina & Pinza fina o madura \\
\hline 18 meses & Sube escaleras & Torres de dos o tres cubos \\
\hline 24 meses & $\begin{array}{l}\text { Baja escaleras y } \\
\text { corre }\end{array}$ & \\
\hline 24-36 meses & Salta en dos pies & \\
\hline $36-48$ meses & Salta en un pie & Ata pasadores \\
\hline
\end{tabular}

Fuente: Salgado $\mathrm{P}^{(6)}$.

Los hitos del desarrollo mencionados son alcanzados en forma variable dentro de un periodo de normalidad. Sin embargo, se han establecido edades aproximadas para que sean aplicadas en forma práctica en el consultorio pediátrico, ya que muchas enfermedades neurológicas pueden tener como primera manifestación una detención o regresión del desarrollo psicomotor.

\section{TRASTORNOS DEL DESARROLLO MOTOR}

En este grupo de enfermedades nos referimos a aquellas condiciones que dificultan la adquisición progresiva de habilidades motoras o que causan detención o regresión de estas. Podemos clasificar estos trastornos en las siguientes categorías: retrasos del desarrollo motor, 
trastornos neurodegenerativos, y trastornos motores de origen central, neuromuscular u osteoarticular ${ }^{(7,9)}$.

Retrasos en el desarrollo motor. Nos referimos a aquellas condiciones de aparición tardía, o no aparición, de alguna o de todas las destrezas motoras. En este punto hay que tomar en cuenta los hitos de desarrollo motor y los rangos de variación entre uno y otro.

Trastornos motores neurodegenerativos. En esta condición hay involución psicomotriz, es decir pérdida de habilidades motoras previamente adquiridas. Principalmente nos referimos a enfermedades de origen metabólico.

Trastornos motores de origen central (SNC). Se incluyen todas las condiciones que ocasionaron una noxa al sistema nervioso central, ocasionando lesiones motoras persistentes adquiridas en época perinatal, natal y posnatal. Se incluyen las siguientes condiciones: lesión cerebral aguda (ejm. traumatismos encéfalo craneanos severos); accidente cerebrovascular; encefalopatía hipóxico-isquémica; infecciones del sistema nervioso central (meningitis, encefalitis, abscesos); leucomalacia periventricular del prematuro; kernícterus, etc. ${ }^{(7,8)}$.

Trastornos motores de origen neuromuscular. Son condiciones que afectan al nervio periférico, a la unión neuromuscular o el músculo, causando principalmente hipotonía con reflejos bajos. Entre los principales trastornos se incluyen la atrofia muscular espinal; la miastenia gravis neonatal; las miopatías congénitas y metabólicas; el hipotiroidismo congénito y el grupo de distrofias musculares ${ }^{(9)}$.

Trastornos motores de origen osteoarticular. Condiciones traumatológicas que causan alteraciones en el desarrollo motor, entre las que se incluyen: luxación congénita de cadera (altera articulaciones de la rodilla y columna); anteversión femoral excesiva; desviaciones de la rodilla, y posiciones viciosas y patológicas del pie.

Existe un último grupo que corresponde a otras enfermedades o situaciones que conllevan una limitación de la actividad motora del organismo donde se incluyen:

Problemas genéticos. Cualquier alteración que involucre el SNC provoca alteración en la motricidad global.

Retardo del desarrollo y retardo mental. Produce lentitud en la adquisición en destrezas y dificultad en la precisión y la armonía de la motricidad.

Alteraciones sensoriales. Influye en la torpeza en su coordinación global y fina.

\section{DESARROLLO SENSORIAL}

\section{DESARROLLO SENSORIAL NORMAL}

El desarrollo sensorial es la base del desarrollo cognitivo motor. Los procesos sensoriales son capacidades que nos permiten relacionarnos con el entorno. Recibimos la información a través de los receptores sensoriales que pueden ser visuales, auditivos o táctiles. Esta información se convierte en sensación para poder organizarla e interpretarla a través de otra habilidad denominada la percepción. Luego, trasmitiremos la información o daremos una respuesta ya sea mediante el llanto, la sonrisa, o la expresión de emociones. De esta forma nos vamos relacionando con nuestro mundo exterior e interior. Si carecemos de estos estímulos o experiencias debido a múltiples factores como las carencias sociofamiliares 0 , lo que es peor, debido a una enfermedad neurológica, se verá afectado el desarrollo en todas sus áreas: motora, emocional, mental, afectiva o social.

Desde las etapas iniciales de la gestación el feto recibe diversos estímulos, tanto del interior como del ambiente exterior. El feto puede percibir los niveles de luz y oscuridad, puede escuchar las voces o los sonidos, o sentir la calidez del útero, puede iniciarse el sentido de la olfacción y del gusto, ya que sentirá el sabor del líquido amniótico que deglute.

\section{DESARROLLO VISUAL}

Es el sistema que proporciona mayor información sobre el mundo exterior. Al nacer, la retina (donde se encuentran los conos y bastones) va a estar completamente desarrollada y es por esto que la percepción de la luz es posible. Mientras que el cristalino aún esta inmaduro, por lo que el enfoque visual estará reducido.

A pesar de que el recién nacido mantiene los ojos cerrados la mayor parte del tiempo, va a fruncir los parpados frente al estímulo de un foco luminoso. El recién nacido es capaz de fijarse en un punto de luz, a pesar de que esta sea borrosa. La visión de colores es restringida, tal vez solo una gama de grises, de baja nitidez. Solo podrá distinguir luz, sombras y movimientos. En el primer mes de vida, mejora la agudeza visual, la cual será nítida a una distancia de 25 a 30 centímetros, justamente la distancia del pecho hasta la cara de la madre. El recién nacido puede mirar la cara de la madre pero no la reconoce hasta los 3 meses de edad, inclusive puede imitar la expresión facial y disfrutar frente a rostros o figuras. El recién nacido presta atención por más tiempo a rostros y círculos concéntricos, por lo que siempre debe evaluarse la preferencia visual al igual que la capacidad de habituarse o deshabituarse frente a un estímulo ${ }^{(3)}$. 
Desde el segundo mes, el bebé ya fija la mirada. Establece la convergencia ocular, sigue objetos en movimiento, no diferencia colores, solo contrastes blancos y negros. Al tercer mes desplaza la mirada de un objeto a otro y reconoce el color rojo. Gira la cabeza siguiendo estímulos interesantes. Descubre su cuerpo, se mira las manos, se interesa por juguetes cercanos. En el cuarto mes ve objetos a distancias variables, percibe detalles pequeños, tiene una capacidad visual cercana al adulto.

Durante los primeros meses de vida; el bebé tendrá incapacidad para reconocer objetos e interpretar los mensajes, van a ser necesarias experiencias repetidas. Por todo esto, es importante la estimulación sensorial.

\section{DESARROLLO AUDITIVO}

Es el sistema más importante para el desarrollo del lenguaje. El estímulo a través de las ondas sonoras ingresará por el conducto auditivo externo, luego al medio y, por último, al oído interno, desde donde serán trasmitidas, mediante un impulso nervioso, por el nervio auditivo hacia la corteza cerebral del lóbulo temporal; donde será procesado el estímulo para la emisión de una respuesta.

El recién nacido es sensible a la intensidad de los sonidos, se sobresalta, incluso desde antes de nacer. No localiza ni dirige su cabeza hacia el estímulo sonoro, prefiere la voz humana ${ }^{(3)}$. Al segundo mes, el bebé localiza mejor la fuente sonora y empieza a interesarse por los sonidos y voces familiares. Desde el tercer mes vuelve la cabeza al sonido, empieza a diferenciar la voz humana de otros sonidos. En el cuarto mes adquiere agudeza y madurez, logrando identificar y localizar la dirección exacta del sonido.

\section{DESARROLLO DEL TACTO, GUSTO Y OLFATO}

El tacto es el sentido con desarrollo más precoz, desde etapas muy tempranas de la gestación. Evoluciona progresivamente desde la séptima semana, cuando empieza a sentir sensaciones en el contorno de la boca, luego en el rostro completo y, finalmente, en ambos pies y el tronco. A las veinte semanas sentirá en todo el cuerpo.

Este sentido es importante no solo para el desarrollo de los reflejos primarios, sino que los receptores ubicados en músculos, tendones, articulaciones y el aparato vestibular, llevaran la información a la corteza cerebral y al cerebelo, para poder percibir nuestro tono muscular, nuestros movimientos, la disposición de nuestros miembros, las partes del cuerpo y la posición en el espacio; todo esto es importante para desarrollar el equilibrio.
Desde el útero las papilas gustativas funcionan, y luego del nacimiento el bebe podrá diferenciar lo dulce de lo ácido y amargo, con preferencia por el sabor dulce. Al cuarto mes; aceptará sabores salados, siempre utilizando el olfato. El recién nacido tiene el olfato desarrollado y podrá diferenciar olores agradables y desagradables, con preferencia por los olores conocidos como el de la madre ${ }^{(4)}$.

Las estructuras básicas del desarrollo sensorial están en el cerebro desde antes del nacimiento. Es necesario perfeccionarlo, establecer redes conectivas a través de experiencias sensoriales, especialmente en los primeros meses de vida. Detectar la deficiencia sensorial es labor primordial del pediatra.

\section{TRASTORNOS DEL DESARROLLO SENSORIAL}

El sentido de la visión permite al niño relacionarse con el entorno e interactuar apropiadamente con él. A partir de los 4 meses de vida la visión dirige al desarrollo motor fino y grueso. El lenguaje también es influido por la exposición a estímulos visuales, asimismo, el desarrollo social depende de la interacción visual, mediante el reconocimiento facial de las emociones. Por lo tanto, es muy importante reconocer tempranamente la disfunción visual e intervenir según corresponda en cada caso. Sospechamos discapacidad visual en un bebé pequeño que no logra enfocar la mirada o seguir rostros. La forma anormal - asimétrica de las pupilas también debe llamar nuestra atención, así como todo movimiento anormal de los ojos. Los preescolares y escolares pueden quejarse de visión borrosa o cefalea. Los niños que ya leen y escriben pueden presentar dificultades en la lectoescritura y/o en el aprendizaje ${ }^{(10)}$.

Asimismo, cuando no es reconocida a tiempo, la discapacidad auditiva lleva a una menor estimulación del niño pequeño y una gran dificultad para desarrollar el lenguaje; además, de una estructuración anómala del pensamiento y consecuencias socioafectivas severas. Si bien se describen una serie de factores de riesgo para discapacidad auditiva (prematuridad, muy bajo peso al nacer, uso de medicamentos ototóxicos, hiperbilirrubinemia neonatal, etc.) se recomienda el tamizaje universal en la edad pediátrica temprana. La sospecha de disfunción auditiva en un bebé aumenta cuando no hay respuestas reflejas al ruido o presenta un lenguaje monótono. Un lactante con hipoacusia no volteará al escuchar su nombre ni intentará localizar sonidos familiares. Cerca del primer año, demorará en adquirir sus primeras palabras y nombrar objetos o personas familiares. Posteriormente, la dificultad se hará más evidente al no lograr entender lo que se le dice, ni contar lo que le pasa. 
Por último, mencionaremos que la disfunción sensorial es un problema común en el niño. Se caracteriza por hipo o hiperrespuesta a determinada información sensorial, y se acompaña con frecuencia de la aparición retardada del lenguaje y de problemas de conducta. Los niños con disfunción sensorial auditiva rechazan los sonidos intensos, angustiándose en ambientes muy ruidosos o con mucha gente. A nivel gustativo y olfativo pueden evitar o rechazar ciertos alimentos y limitar así la ingesta adecuada. Muchas veces estos menores, además, tienen dificultades en los sentidos de la propiocepción y el equilibrio; y presentan hiperlaxitud articular. Los infantes con disfunción sensorial pueden presentar menores niveles de atención y concentración, niveles de actividad muy altos o muy bajos, dificultades en la coordinación y planeamiento del movimiento, dificultad para interactuar con sus pares y baja autoestima. Todo ello requiere un reconocimiento temprano y una intervención oportuna.

\section{DESARROLLO DEL LENGUAJE}

\section{DESARROLLO NORMAL DEL LENGUAJE}

El lenguaje es un fenómeno cultural y social que usa símbolos y signos adquiridos, los cuales permiten la comunicación con los demás. Esta es una destreza que se aprende naturalmente y se convierte en pieza fundamental de la comunicación puesto que admite proyectar emociones, pensamientos e ideas en el tiempo y en el espacio. El lenguaje oral constituye el principal (y a veces el único) medio de información y cultura, por tanto, es un factor importante de identificación a un grupo social (11).

En el niño podemos reconocer las siguientes formas de lenguaje: el lenguaje gestual, con recepción por la vía visual y emisión a través de gestos o muecas faciales y manuales (de 0 a 12 meses); el lenguaje verbal, con recepción por vía auditiva y emisión a través del habla (de 1 a 5 años), y el lenguaje escrito, con recepción visual por medio de la lectura y emisión a través de la escritura (más allá de los 5 años) (11,15).

Las teorías vocales sostienen que el lenguaje evolucionó a partir de un amplio grupo de llamadas instintivas que expresaban estados emocionales tales como angustia, júbilo y excitación sexual. También se ha planteado que el lenguaje haya surgido de la evolución conjunta de los gestos y la vocalización, lo que podría justificar la inexplicable correlación entre la dominancia manual y el lenguaje verbal y de signos, ambos localizados en el hemisferio izquierdo ${ }^{(11,15)}$. En la adquisición del lenguaje distinguimos inicialmente la etapa preverbal, que ocurre durante los primeros 10 a 12 meses de edad. Otros la consideran como la etapa del nivel fónico puro, debido a que el infante emite solo sonidos onomatopéyicos.

Durante esta etapa, la comunicación que establece el niño es con su medio familiar, especial y particularmente con su madre, y es de tipo afectivo y gestual. Para estimularlo lingüísticamente la madre puede utilizar, junto con el lenguaje afectivo y gestual, el lenguaje verbal. La palabra debe acompañar siempre al gesto y a las actividades de la madre con su hijo ${ }^{(13)}$.

La etapa lingüística se inicia con la expresión de la primera palabra. No se puede decir con precisión cuándo comienza. Por eso, la fecha de su aparición está diversamente fijada, ya que los estudios al respecto se basan mayormente en las informaciones que dan las madres. Los diferentes especialistas estiman que el $90 \%$ de los niños que van a hablar, dicen sus primeras palabras entre los 15 a 18 meses ${ }^{(13)}$.

Para la adquisición apropiada del lenguaje, el niño requiere la integridad de los órganos de la respiración (pulmones, músculos costales y el diafragma, necesarios para la emisión de sonidos); de los órganos de la fonación (laringe y las cuerdas vocales, que se emplean en la producción de la voz); los órganos de resonancia (faringe, boca y fosas nasales, que modulan el tono de los sonidos), y de los órganos de la articulación (paladar, lengua, mandíbulas, labios y dientes) que modularán el tono de la voz ${ }^{(11)}$.

El desarrollo del lenguaje dependerá de la interacción de diferentes factores, entre los cuales se encuentran las relaciones afectivas e intelectuales del niño, quien debe sentirse emocionalmente seguro y lingüísticamente estimulado; la personalidad del niño y de los adultos que lo rodean; la maduración biológica (del sistema nervioso, auditivo, aparato fonador e inteligencia), y de los propios procesos de aprendizaje. Pocos conocemos la secuencia de desarrollo social y del lenguaje, lo que motiva que muchos niños con retraso en estas áreas, sean referidos después de los dos años de edad, lo cual implica la pérdida del período crítico para el desarrollo social y del sistema auditivo y del habla, que está comprendido entre los 6 y 24 meses de edad (Tabla 2). Finalmente, debemos recordar que la participación del lenguaje en el aprendizaje pedagógico es tan fundamental, que cualquier limitación en su adquisición tiende a afectar la capacidad del aprendizaje escolar ${ }^{(11)}$.

\section{TRASTORNOS DEL LENGUAJE}

La calidad del lenguaje depende de una adecuada estructura anátomofuncional y de la influencia del medio. En el hogar, el niño debe estar rodeado de personas con lenguaje más avanzado, comprometidas afectivamente 
Tabla 2. Desarrollo cronológico del lenguaje

\begin{tabular}{ll}
\hline \multicolumn{1}{c}{ Edad } & \multicolumn{1}{c}{ Características } \\
\hline Recién nacido & Llanto \\
3 meses & Gutureo, gorjeo, vocalizaciones \\
6 meses & Silabeo \\
9 meses & Balbuceo (bisilábico) \\
12 meses & Palabreo \\
18 meses & Parloteo (intentos de hablar corrido) \\
24 meses & Fraseo (une dos palabras) \\
30 meses & Oraciones \\
\hline
\end{tabular}

Fuente: Huanca D ${ }^{(11)}$.

con el niño en actividades conjuntas, que estimulen en la fase de aprendizaje el juego interactivo entre ellos. El desarrollo del lenguaje debe darse sobre una base afectiva que cree vínculos de relación entre el niño y los adultos relevantes de su entorno. El niño con trastorno del lenguaje puede presentar desarrollo deficiente de la comprensión (habilidad para entender o decodificación) o de la producción (capacidad de lograr una comunicación simbólica hablada, escrita o gestual) (Tabla 3). Podemos encontrar algún trastorno de la audición o del lenguaje receptivo, del habla o del lenguaje expresivo, aunque son frecuentes los trastornos mixtos. En la cascada asociada al retraso del lenguaje se encuentran alteraciones del desarrollo social e intelectual, aislamiento y/o regresión, rendimiento académico pobre, y finalmente problemas de aprendizaje y sociales; por lo tanto, previniendo el retraso del lenguaje, se prevendrá esta cascada ${ }^{(12,13)}$.

La prevalencia de los trastornos del lenguaje llega al $15 \%$ en los preescolares, 3 a $6 \%$ en los escolares. Es cuatro veces más común en varones, y también es frecuente la historia familiar. Los trastornos de expresión de lenguaje son notorios entre los 18 y 36 meses ${ }^{(14)}$.

Tabla 3. Signos de alarma en la adquisición del lenguaje

\begin{tabular}{|c|c|}
\hline Edad & Signos de alarma \\
\hline $0-1$ mes & Llanto raro \\
\hline 2-4 meses & Ausencia de sonrisa social \\
\hline 6 meses & No vocaliza ni balbucea \\
\hline 9 meses & No silabea \\
\hline 12 meses & Pierde habilidades \\
\hline 15 meses & No señala, no utiliza tres palabras \\
\hline 18-24 meses & $\begin{array}{l}\text { No sigue instrucciones simples, no dice } \\
\text { "mamá" u otros nombres, no reconoce } \\
\text { partes de su cuerpo }\end{array}$ \\
\hline 24-36 meses & $\begin{array}{l}\text { No dice frases de dos palabras, no sigue } \\
\text { instrucciones de dos pasos }\end{array}$ \\
\hline $36-48$ meses & $\begin{array}{l}\text { Uso incorrecto de palabras, sustituye una } \\
\text { por otra }\end{array}$ \\
\hline 4-6 años & No habla correctamente \\
\hline
\end{tabular}

Fuente: Moreno-Flagge $\mathrm{N}^{(12)}$.
En caso de que el niño tarda en hablar, debemos considerar las siguientes etiologías: retraso simple del lenguaje (RSL), trastorno específico del lenguaje (TEL), trastornos del espectro autista (TEA), discapacidad intelectual, hipoacusia, hijos de padres sordo-mudos y privación ambiental extrema. La hipoacusia es la tercera causa en frecuencia, por lo que se debe descartar, en primer lugar, en todos los niños con retraso o alteraciones en el lenguaje. Su incidencia en el período neonatal es de 3-5/1000 nacidos vivos, siendo la enfermedad congénita más frecuente, incluso más que el hipotiroidismo ${ }^{(15)}$.

En los niños que dejan de hablar (regresión del lenguaje), debemos buscar afasias adquiridas secundarias a lesiones cerebrales (expresivas o receptivas); síndrome de Landau Kleffner (afasia epiléptica); mutismo selectivo; regresión autista; síndrome de Rett; trastorno desintegrativo infantil (TDI), y enfermedades degenerativas $(12,14,15)$.

Cuando el niño habla mal, reconoceremos alguno de estos trastornos: tartamudez (también llamada espasmofemia), disartria, dislalia, trastornos de la prosodia y voz nasal. Debemos distinguir entre la tartamudez evolutiva, parte de la adquisición normal del lenguaje, y la verdadera, que requiere intervención ${ }^{(12,14,15)}$.

Otros trastornos incluyen el de tipo semánticopragmático y los trastornos del lenguaje escrito (dislexia, disgrafia, hiperlexia, etc.) que requieren reconocimiento y una intervención temprana y oportuna.

\section{DESARROLLO SOCIAL}

\section{DESARROLLO SOCIAL NORMAL}

Un recién nacido es capaz de mirar y fijar la mirada en los ojos de las personas, en especial de su madre. A los 3 meses logra la sonrisa social; a los 6 meses ya tiene risa social: mira a los ojos, sonríe y se ríe espontáneamente en presencia de personas (sin estimulación táctil o sin cosquillas), no es la risa refleja frente a objetos o animales. Alza las manos, le gusta que lo carguen.

A los nueve meses imita, hace adiós con la mano, imita gestos faciales, siempre mirando a los ojos. Juega a taparse con el pañal. Fijando la mirada en una persona, gruñe, grita, "chilla", mueve las manos cuando quiere algo (gesto protoimperativo). Toca su imagen en el espejo, entiende el "no". Estira los brazos para que lo carguen. Presenta ansiedad o angustia ante los extraños (Ilora cuando se acerca un extraño o familiar al que no ha visto por algunos días). Poco tiempo después, a los 12 meses, señala; fija la mirada en una persona, 
estira el brazo y señala lo que quiere (verbaliza, grita y establece contacto visual alternativamente entre el objeto y la persona con la única intención de dirigir la atención de la persona hacia el objeto que quiere (gesto protodeclarativo). Responde a su nombre, demuestra afecto, abraza y le gusta que lo abracen, apoya su cara en otra cara, sonríe y ríe ${ }^{(16)}$.

Llegados los 15 meses responde cuando lo llaman por su nombre (verbal o visualmente), a los 18 meses trae objetos para mostrarlos. Señala partes de su cuerpo. Señala lo que quiere, fija la mirada, sonríe y se ríe. A los 24 meses disminuye la ansiedad ante los extraños, se interesa por otros niños, quiere jugar con ellos. Imita tareas domésticas. Tiene imaginación y desarrolla el juego simbólico. Estos gestos sociales, una vez desarrollados, se mantienen en menor o mayor grado a lo largo de toda la vida, empleándose cada vez que interactuamos con otras personas (Tabla 4).

Tabla 4. Desarrollo cronológico social normal

\begin{tabular}{ll}
\hline \multicolumn{1}{c}{ Edad } & \multicolumn{1}{c}{ Gestos sociales } \\
\hline Recién nacido & Mira \\
3 meses & Sonríe \\
6 meses & Ríe \\
9 meses & Imita \\
12 meses & Señala \\
15 meses & Voltea \\
18 meses & Muestra \\
24 meses & Juega \\
\hline
\end{tabular}

Fuente: Huanca D ${ }^{(16)}$.

\section{TRASTORNOS DEL DESARROLLO SOCIAL Y AUTISMO}

El vocablo autismo está presente en la literatura médica desde 1911, y es en 1943 cuando Leo Kanner y Hans Asperger realizan las primeras descripciones relevantes. Kanner describió niños con aislamiento profundo, que mostraban deseo obsesivo de no aceptar cambios, que solían presentar una fisionomía inteligente y pensativa, y su conducta se caracterizaba por una relación intensa con objetos y una comunicación verbal anormal. Estos criterios diagnósticos fueron modificados por Asperger en 1944, quien agregó la falta de empatía, ingenuidad, poca habilidad para hacer amigos, lenguaje pedante y repetitivo, pobre comunicación no verbal, interés desmesurado por ciertos temas; torpeza motora y mala coordinación.

La definición del autismo ha evolucionado en los últimos años, hasta llegar a la que tenemos actualmente y que corresponde al Diagnostic and Statistical Manual of Mental Disorders, Fifth Edition (DSM-5) (17) (Tabla 5). Los criterios utilizados han sido resumidos en dos puntos principales: déficit en la interacción social y la
Tabla 5. Criterios diagnósticos del trastorno del espectro del autismo DSM-5

A. Déficits persistentes en comunicación social e interacción social a lo largo de múltiples contextos, según se manifiestan en los siguientes síntomas, actuales o pasados.

1. Déficits en reciprocidad socioemocional; rango de comportamientos que, por ejemplo, van desde mostrar acercamientos sociales inusuales y problemas para mantener el flujo de ida y vuelta normal de las conversaciones; a una disposición reducida por compartir intereses, emociones y afecto, a un fallo para iniciar la interacción social o responder a ella.

2. Déficits en conductas comunicativas no verbales usadas en la interacción social; rango de comportamientos que, por ejemplo, van desde mostrar dificultad para integrar conductas comunicativas verbales $y$ no verbales a anomalías en el contacto visual y el lenguaje corporal, o déficits en la comprensión y uso de gestos a una falta total de expresividad emocional o de comunicación no verbal.

3. Déficits para desarrollar, mantener y comprender relaciones; rango de comportamientos que van, por ejemplo, desde dificultades para ajustar el comportamiento para encajar en diferentes contextos sociales a dificultades para compartir juegos de ficción o hacer amigos hasta una ausencia aparente de interés en la gente.

B. Patrones repetitivos y restringidos de conductas, actividades e intereses, que se manifiestan en, al menos, dos de los siguientes síntomas, actuales o pasados.

1. Movimientos motores, uso de objetos o habla estereotipados o repetitivos (ej., movimientos motores estereotipados simples, alinear objetos, dar vueltas a objetos, ecolalia, frases idiosincrásicas).

2. Insistencia en la igualdad, adherencia inflexible a rutinas o patrones de comportamiento verbal y no verbal ritualizado (ej. malestar extremo ante pequeños cambios, dificultades con las transiciones, patrones de pensamiento rígidos, rituales para saludar, necesidad de seguir siempre el mismo camino o comer siempre lo mismo).

3. Intereses altamente restringidos, obsesivos, que son anormales por su intensidad o su foco (ej. apego excesivo o preocupación excesiva con objetos inusuales, intereses excesivamente circunscritos o perseverantes)

4. Hiper o hiporreactividad sensorial o interés inusual en aspectos sensoriales del entorno (ej. indiferencia aparente al dolor/temperatura, respuesta adversa a sonidos o texturas específicas, oler o tocar objetos en exceso, fascinación por las luces u objetos que giran).

C. Los síntomas deben estar presentes en el período de desarrollo temprano (aunque pueden no manifestarse plenamente hasta que las demandas del entorno excedan las capacidades del niño, o pueden verse enmascaradas en momentos posteriores de la vida por habilidades aprendidas).

D. Los síntomas causan alteraciones clínicamente significativas a nivel social, ocupacional o en otras áreas importantes del funcionamiento actual.

E. Estas alteraciones no se explican mejor por la presencia de una discapacidad intelectual (trastorno del desarrollo intelectual) o un retraso global del desarrollo. La discapacidad intelectual y el trastorno del espectro de autismo con frecuencia coocurren, para hacer un diagnóstico de comorbilidad de trastorno del espectro de autismo y discapacidad intelectual, la comunicación social debe estar por debajo de lo esperado en función del nivel general de desarrollo.

Fuente: DSM-5. Manual diagnóstico y estadístico de los trastornos mentales. 2014 (17) 
comunicación, y presencia de intereses restringidos y repetitivos (comportamientos estereotipados verbales o motoras, sensoriales o comportamientos inusuales, y/o adhesión excesiva a rutinas y patrones ritualizados de comportamiento).

El diagnóstico es fundamentalmente clínico, no existe ningún examen biológico que pueda validarlo. Presenta manifestaciones que van desde un completo desinterés por otras personas hasta la repetición de preguntas para mantener la interacción social, son niños muy distantes que evaden la mirada, o se acercan demasiado; tocando, besando, oliendo inapropiadamente. Debe sospecharse en niños alrededor de los seis meses de vida, algunos de los síntomas sugestivos son la dificultad en la alimentación, en el intercambio de miradas e hipotonía. Se observa un bebe que nunca llora o no se molesta, irritable a pequeños estímulos.

No existe una causa única conocida para el autismo. Existen múltiples teorías que intentan explicar esta enfermedad, dentro de las cuales tenemos a la teoría psicógena, genética, epigenética, estructural, metabólica, histológica, bioquímica e infecciosa; sin embargo, la teoría genética es la que actualmente tiene mayor validez.
Una vez reconocido el problema, se debe emplear un enfoque interdisciplinario y multidisciplinario, con comunicación constante entre psiquiatras, psicólogos, neurólogos, terapistas y educadores. Es necesario precisar los criterios diagnósticos y de sospecha entre profesionales, padres y educadores para un diagnóstico certero y oportuno.

\section{CONCLUSIONES}

Hemos presentado una visión sucinta de las características y evolución del neurodesarrollo infantil normal, con la finalidad de identificar oportunamente la presencia de anomalías en una o varias de sus áreas, ya sea motora, motora fina, sensorial, del lenguaje o social. Ante la detección o sospecha de alteración del desarrollo neurológico, todo pediatra o profesional de la salud que atiende niños deberá alertar oportunamente a los padres para la búsqueda de la terapia correspondiente.

Contribuciones de autoría: Todos los autores contribuyeron con la redacción del artículo. MPM y SMVS contribuyeron con la concepción y estructuración del mismo.

Fuentes de financiamiento: ninguna.

Conflictos de interés: los autores declaran no tener conflictos de interés.

\section{REFERENCIAS BIBLIOGRÁFICAS}

1. Victora CG, Horta BL, Loret de Mola C, Quevedo L, Tavares R, Gigante D, et al. Association between breastfeeding and intelligence, educational attainment, and income at 30 years of age: a prospective birth cohort study from Brazil. Lancet Global Health 2015;3(4):e199-205.

2. Douglas-Escobar M, Elliot E, Neu J. Effect of intestinal microbial ecology on the developing brain. JAMA Pediatr 2013;167(4): 374-9. doi: 10.1001/ jamapediatrics.2013.497.

3. Volpe J. Neurology of the newborn infant, 5th ed. Philadelphia: Saunders Elsevier; 2008.

4. Walker SP, Wachs TD, GranthamMcGregor S, Black M, Nelson C, Huffman $S$, et al. Inequality in early childhood: risk and protective factors for early child development. Lancet. 2011;378(9799):1325-38. doi: 10.1016/S0140-6736(11)60555-2.

5. Illingworth R. El desarrollo normal del lactante y el niño. 1972. Williams \& Wilkins.
6. Salgado P. Desarrollo motor normal. Análisis desde el enfoque del Neurodesarrollo, Santiago de Chile: Universidad de Chile; 2007.

7. Elsevier España. Parálisis cerebral infantil. Anales de Pediatría Continuada. 2005;3(2):73-8.

8. Póo Argüelles P. Parálisis cerebral infantil. Madrid: Asociación Española de Pediatría; 2008.

9. Prats Viñas J. Enfoque diagnóstico del niño hipotónico. Baracaldo: Hospital Universitario de Cruces. 2008.

10. Fenichel GM. Clinical pediatric neurology: a signs and symptoms approach, 6th ed. Shreveport: Elsevier Saunders; 2009.

11. Huanca Payehuanca D. Desarrollo del lenguaje. Rev Peru Pediatr. 2008;61(2):98-104.

12. Moreno-Flagge N. Trastornos del lenguaje: Diagnóstico y tratamiento. Rev Neurol. 2013;57(Supl 1): S85-94.

13. Barragán E, Lozano S. Identificación temprana de trastornos dellenguaje. Rev Med Clin Condes. 2011;22(2):227-32.
14. Gallego C, Rodríguez-Santos F. Trastornos específicos del lenguaje. Curso de actualización Pediatría. Madrid: Exlibris Ediciones. 2009. p 239-48.

15. Artigas J, Rigau E, García-Nonell K. Trastornos del lenguaje. AEP: Protocolos de actualización 2008, 24:178-84.

16. Huanca Payehuanca D. Desarrollo social en niños. Rev Peru Pediatr. 2008;61(2):133-8.

17. American Psychiatric Association. DSM-5. Manual diagnóstico y estadístico de los trastornos mentales, 5th ed. Buenos Aires: Panamericana; 2014.

Correspondencia: Dra. María del Pilar

Medina

Dirección: Instituto Materno Perinatal Av. Miró Quesada 941 - Lima, Perú. Teléfono: (+511) 987709754

Correo electrónico:mpilarmedinaa@gmail.com 